Rev Biomed 2006; 17:195-211.

\title{
Tratamiento de la enfermedad hepática crónica inducida por el virus de la hepatitis $C$ : bases moleculares y celulares.
}

Revisión

Angel León-Buitimea ${ }^{1}$, Alfonso Leija-Salas ${ }^{1}$, Germán Garrido-Fariña², Jorge Reyes-Esparzaํㅡㄹ Lourdes Rodríguez-Fragoso ${ }^{1}$.

${ }^{1}$ Facultad de Estudios Superiores Cuautitlán, Universidad Autónoma de México (UNAM). Estado de México, México. ${ }^{2}$ Facultad de Farmacia. Universidad Autónoma del Estado de Morelos (UAEM). Cuernavaca, Morelos, México.

\section{RESUMEN.}

En esta revisión se analiza el estado actual del tratamiento de la enfermedad hepática crónica inducida por el virus de la hepatitis C. El presente trabajo constituye una revisión de los fármacos empleados a nivel experimental y en humanos para el tratamiento de la enfermedad hepática crónica en los últimos 10 años, así como también se hace referencia a las tendencias actuales para abordar tal padecimiento. El principal obstáculo del desarrollo de terapias antifibróticas es la lenta evolución de la fibrosis, la cual toma años e inclusive décadas en el hombre. Los avances actuales en el conocimiento de la patogénesis molecular de la fibrosis hepática y el papel de la célula estelar activada están proporcionando las bases para el desarrollo de nuevas terapias. (Rev Biomed 2006; 17:195-211)

Palabras clave: hepatitis C, fibrosis, célula estelar hepática.

\section{SUMMARY.}

Treatment of chronic liver disease induced by the hepatitis $C$ virus: Molecular and cellular bases.

In this review we analyse the currently available therapies for liver fibrosis induced by the hepatitis $\mathrm{C}$ virus. The present work is a review about the drugs employed at an experimental level and in the human beings for the treatment of chronic liver diseases in the last ten years. It also refers to the actual trends for solving this problem. The major obstacle to antifibrotic drug development is the slow evolution of fibrosis, which takes years or even decades in man. Recent insights into the molecular pathogenesis of hepatic fibrosis and the role of activated hepatic stellate cells provide hope for the future development of successful therapies.

(Rev Biomed 2006; 17:195-211)

Key words: Hepatitis C, fibrosis, hepatic stellate cell.

\section{INTRODUCCIÓN.}

La infección por el virus de la hepatitis C (VHC) se ha convertido en un grave problema de salud a nivel mundial. Se estima que 170 millones 


\section{A León-Buitimea, A Leija-Salas, G Garrido-Fariña, J Reyes-Esparza, L Rodríguez-Fragoso}

de personas en el mundo están infectadas con el virus de hepatitis C. Sólo en Estados Unidos hay 4 millones de personas infectadas, y en México 1.5 millones (1). En la actualidad esta enfermedad representa la causa principal de trasplante hepático como manifestación de enfermedad hepática terminal.

La evolución natural de la hepatitis C crónica es todavía objeto de debate debido a que la infección tiene un curso silencioso, prácticamente asintomático en la mayoría de los pacientes y, en consecuencia, el diagnóstico se hace cuando la enfermedad ya es crónica. Veinte por ciento de los pacientes con hepatitis $C$ crónica desarrollarán cirrosis a 20 años de adquirir la infección (2-4). Una preocupación real es la cirrosis hepática crónica secundaria al VHC y su pronóstico, ya que en el seguimiento de cinco años la sobrevida corresponde a tan sólo $50 \%$. De igual manera, existe el riesgo de desarrollar otra temida complicación, el carcinoma hepatocelular (CHC) $(5,6)$. La importancia de la cirrosis no es solamente por el hecho de que muchas personas mueren, sino porque la mortalidad debida a cirrosis se está incrementando y la mayoría de los pacientes son adultos en edad productiva. Dado el curso tan lento de la enfermedad, el número de casos de cirrosis y sus complicaciones se ha elevado y se estima que seguirá elevándose en las siguientes dos décadas (7). En consecuencia, resulta evidente la importancia clínica de contar e instituir un tratamiento efectivo con el propósito de modificar este curso clínico. En esta revisión se analiza el estado actual del tratamiento de la enfermedad hepática crónica de origen viral.

\section{Fibrosis hepática.}

La fibrosis hepática es una acumulación reversible de componentes de la matriz extracelular en el parénquima hepático como una reacción al daño crónico. Una característica de la enfermedad hepática crónica es el desarrollo de la fibrosis hepática. Los cambios fibróticos durante la enfermedad hepática crónica son causados por una pérdida de los mecanismos homeostáticos, los cuales bajo circunstancias fisiológicas controlan no sólo la producción y depósito de la matriz (fibrogénesis) sino también su degradación y remoción (fibrolisis) (8).

La fibrogénesis representa una compleja reacción fisiopatológica en la cual participan las células parenquimatosas y no parenquimatosas, células inflamatorias, los componentes de la matriz extracelular y varios mecanismos de transferencia de señales autócrinos, parácrinos y yuxtácrinos (9-13). La fibrogénesis es regulada por muchos factores, los cuales influyen en su inicio y duración, y ésta culmina con el desarrollo de fibrosis. En términos generales, la fibrosis se caracteriza por:

1) Incrementos de 3 a 6 veces de la mayoría de las moléculas de la matriz extracelular (MEC) de naturaleza colagenosa y no colagenosa $(14,15)$.

2) Elevación desproporcionada de algunos componentes individuales de la MEC, incluyendo varios tipos de colágena, proteoglucanos y glicoproteínas estructurales (16).

3) Pequeños cambios en la microcomposición de moléculas específicas de la MEC, por ejemplo, el grado de hidroxilación de la cadena alfa de la colágena, el grado de sulfatación de los glucosaminoglucanos, etc. (17).

4) Redistribución de la MEC en el hígado dañado conduciendo al depósito subendotelial del tejido conectivo en el espacio de Disse (fibrosis perisinusoidal) (18).

La fibrosis hepática se origina no solamente como una consecuencia de los cambios en la secreción de la matriz sino de cambios en su degradación, lo cual significa que en la enfermedad hepática crónica hay una pérdida del balance funcional dinámico entre la fibrogénesis y la fibrolisis.

\section{Bases moleculares y celulares de la fibrosis hepática.}

La fibrosis hepática es un proceso de reparación-cicatrizante que ocurre cuando el hígado

\section{Revista Biomédica}


Tratamiento de la enfermedad hepática crónica

es dañado crónicamente y, como ya señalamos, se caracteriza por excesivo depósito de matriz extracelular en el tejido hepático. En esencia, las reacciones extensamente interrelacionadas que llevan a la fibrosis hepática son las siguientes: daño a las células parenquimatosas (hepatocitos), el cual parece ser (con algunas excepciones) el evento iniciador y perpetuador, seguido por inflamación, regeneración hepatocelular, producción de matriz extracelular y desorganización de la arquitectura lobular (19-22).

Los hepatocitos dañados como consecuencia de la invasión del virus de hepatitis $\mathrm{C}$ generan una reacción inflamatoria (2). Esos hepatocitos junto con el infiltrado de células inflamatorias activan a las células de Kupffer (23). La activación de las células de Kupffer libera un sinnúmero de factores solubles, incluyendo citoquinas, y entre ellas el factor de crecimiento transformante beta (TGF- $\beta$ ), el factor de crecimiento derivado de plaquetas (PDGF), el factor de necrosis tumoral alfa (TNF- $\alpha$ ), especies oxígeno reactivas (ROS) y otros factores (24-27). Todos estos factores actúan sobre la célula estelar hepática (HSC, células acumuladoras de grasa o células de Ito) que están localizadas en el espacio perisinusoidal y cuya función es almacenar la mayoría de la vitamina A del organismo. Las células estelares hepáticas son normalmente quiescentes y producen pequeñas cantidades de componentes de matriz extracelular, tales como la laminina y colágena tipo IV para la formación de la membrana basal (28). Cuando ellas son expuestas a factores solubles provenientes de hepatocitos dañados y de células de Kupffer activadas, la célula estelar pierde su contenido de lípido y sufre una transformación morfológica que semeja a un miofibroblasto. Esta transición se conoce como activación de la célula estelar (ver figura 1).

El daño celular es el evento iniciador, donde las células de Kupffer junto con la participación de otros tipos de células no parenquimatosas (células

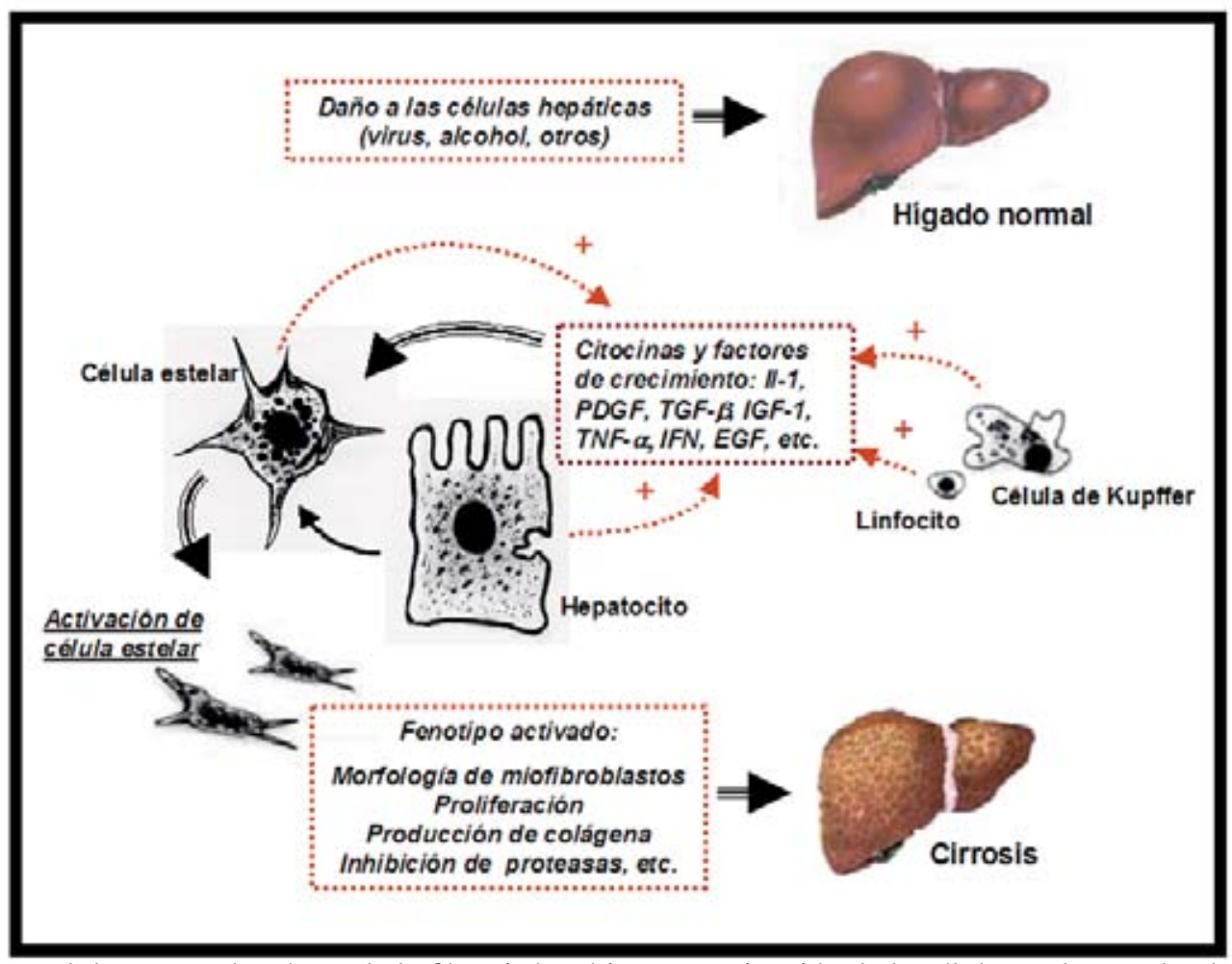

Figura 1.- Bases celulares y moleculares de la fibrosis hepática. La activación de la célula estelar resulta de su interacción con las diferentes células residentes en el hígado a través de factores de crecimiento e interleucinas que actúan en forma parácrina y autócrina. 


\section{A León-Buitimea, A Leija-Salas, G Garrido-Fariña, J Reyes-Esparza, L Rodríguez-Fragoso}

endoteliales, células pit, células de conductos biliares y fibroblastos) son los mediadores. Mientras que las células estelares actúan como efectores, como consecuencia de la comunicación química cruzada entre los diferentes tipos celulares, mediada por diferentes factores solubles (29-31).

\section{Activación de la célula estelar.}

En el tejido hepático normal las células estelares están involucradas en muchas funciones homeostáticas del hígado incluyendo el almacenamiento de la vitamina A, la regulación del flujo sanguíneo sinusoidal, la comunicación con hepatocitos, el mantenimiento del fenotipo del hepatocito, la remodelación y el depósito de la matriz extracelular, y la regulación de la inflamación local. Sin embargo, se sabe que en el hígado dañado la célula estelar es el blanco primario para el estímulo inflamatorio y como resultado de esto produce diversos componentes de la matriz extracelular (32).

Muchos factores contribuyen a la activación de la célula estelar. El daño hepatocelular, como señalamos previamente, es el factor primario y continuador que lleva a la activación de esta célula. La activación de la célula estelar sucede en sitios de inflamación y necrosis de células hepáticas. El inicio del proceso de activación in situ se considera como el evento patogénico clave en la iniciación de la fibrogénesis. El proceso de activación de la célula estelar incluye los siguientes eventos:

1.- La estimulación de la proliferación de la célula estelar, por acción del TNF (33).

2.- La transdiferenciación fenotípica (frecuentemente llamada transformación) vía transición de las células estelares "almacenadoras" de retinoides al fenotipo "sintético" (miofibroblasto). La célula estelar se caracteriza por tener una menor densidad de gotas de grasa que contienen vitamina $\mathrm{A}$, un retículo endoplásmico hipertrofiado, abundante desmina (en rata, pero no en humanos), gelsolina, y filamentos de $\alpha$ - actina de músculo liso (34).

3.- La expresión génica, síntesis y secreción de un amplio espectro de proteínas de matriz extracelular (colágenas, glicoproteínas y proteoglucanos) (35).

4.- La expresión génica, síntesis y secreción de citoquinas pro-inflamatorias y pro-fibrogénicas, quimocinas, y factores de crecimiento (36).

5.- La activación de la contractilidad (en respuesta a endotelina 1, angiotensina II trombina, y eicosanoides), lo cual sugiere que la célula estelar activada también adquiere funciones vasoreguladoras en el hígado dañado $(27,31)$.

Intensas investigaciones se han realizado para identificar los mediadores fibrogénicos que estimulan la activación de la célula estelar durante el proceso de fibrogénesis, y en consecuencia la amplificación de la producción de la matriz extracelular en la enfermedad hepática crónica. Entre las reacciones celulares que se han identificado y que se sabe participan en la activación de la célula estelar se incluyen:

1. La expansión de la poza de macrófagos debido a la proliferación local de células de Kupffer y el influjo de monocitos, junto con la activación celular y producción de numerosos mediadores fibrogénicos.

2. La adhesión, influjo, y desintegración de plaquetas y liberación de factores de crecimiento en el sitio del daño.

3. El daño a células endoteliales sinusoidales y liberación de mediadores fibrogénicos.

4. La inactivación, dependiente del daño, de un inhibidor de la proliferación de la célula estelar derivado de hepatocitos y la liberación de mediadores fibrogénicos por hepatocitos dañados.

Los cambios en el microambiente de la célula estelar hepática debidos a una matriz extracelular y una composición celular extensamente alterada implican que los hepatocitos, las células de Kupffer y las plaquetas agregadas, representan las principales células efectoras que liberan mediadores estimulando en una vía parácrina a las células estelares y a los miofibroblastos (11, 19 y 22). La figura 2 muestra los cambios microscópicos

\section{Revista Biomédica}


Tratamiento de la enfermedad hepática crónica

A

B

C

$\mathrm{D}$

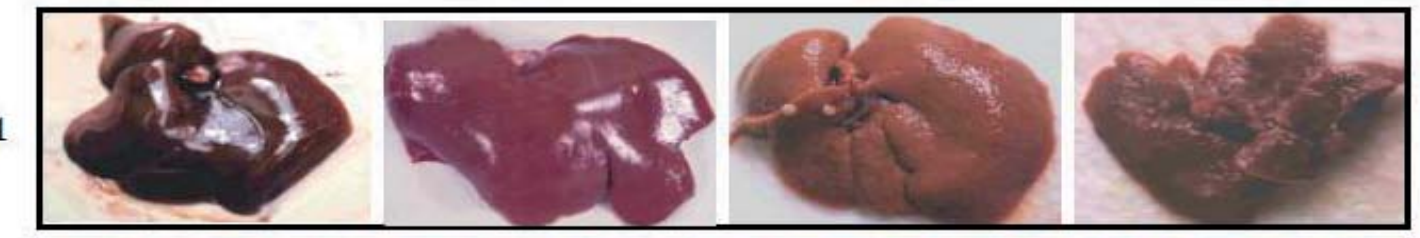

2

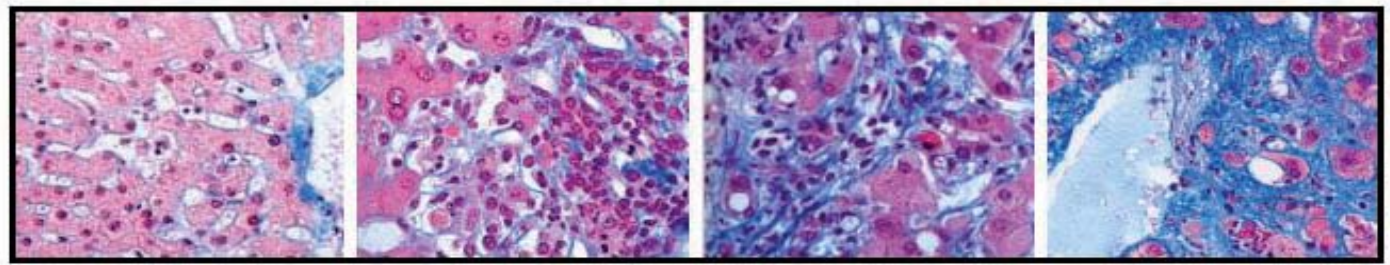

3

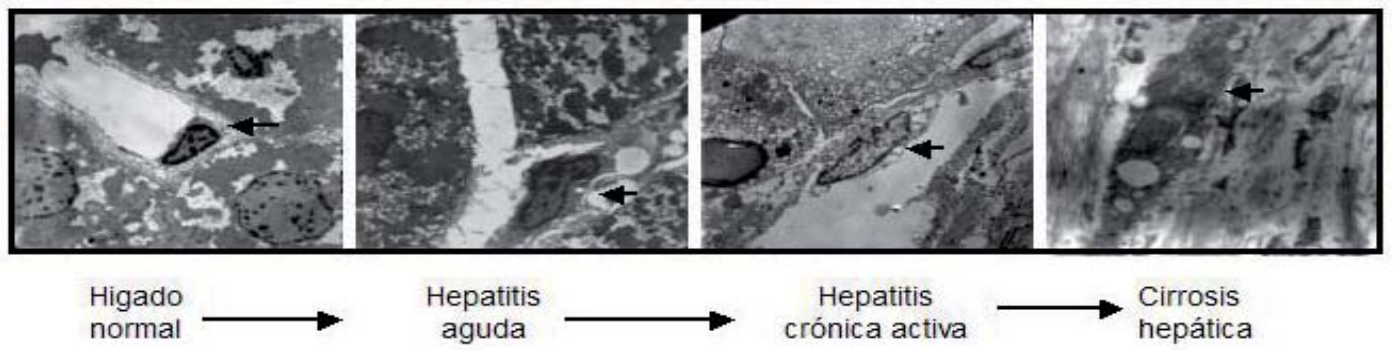

Figura 2.- Desarrollo de la cirrosis hepática: el daño al hígado normal (A) resulta en inflamación y activación de la célula estelar (flechas) con la subsiguiente proliferación y producción de matriz extracelular(B); esto conlleva al desarrollo de fibrosis (C), y finalmente a cirrosis (D). (1) Imagen macroscópica del hígado; (2) Corte histológico del hígado (tinción Tricrómica de Mason X260); (3) cambios fenotípicos de la célula estelar durante el daño hepático (3A, 3B, y 3C, microfotografías X3000) (3D, microfotografía X 7000).

y macroscópicos que sufre el hígado durante la evolución de la fibrosis hepática.

\section{Consecuencias clínicas de la fibrosis y cirrosis hepática.}

Característicamente, la presentación de la hepatitis viral aguda es asintomática. Posterior a los seis meses siguientes a la infección inicial la enfermedad es indetectable. La mayoría de los síntomas comunes comienzan después de algunos años de la infección inicial. En este tiempo el paciente presenta comúnmente fatiga y puede presentar otros síntomas que incluyen fiebre, dolor en los músculos y articulaciones, náusea, vómito, pérdida del apetito, dolor abdominal vago y algunas veces diarrea. Una minoría de pacientes notan orina oscura y heces ligeramente coloridas seguido por ictericia, en la cual la piel y conjuntiva aparecen amarillas.

Sin embargo, la evolución de la hepatitis viral crónica es muy desfavorable. Se ha observado en personas con hepatitis aguda postransfusional o esporádica, que sólo en el 15-30\% existe resolución espontánea de la anormalidad. Del 70 al 85\% de los individuos muestran persistencia del virus, lo que en la mayoría de los casos se manifiesta en la forma de hepatitis crónica. Veinte por ciento de esos enfermos desarrolla cirrosis hepática en un periodo aproximado de 30 años después de la infección. Los pacientes frecuentemente no muestran anormalidades funcionales. Sin embargo, hay el caso de aquellos pacientes con enzimas hepáticas elevadas que presentan inflamación intralobular, acompañada de infiltración celular mononuclear, fibrosis y expansión del tracto portal y necrosis hepatocelular $(2,7)$. La mayoría de los pacientes portadores del VHC y con enfermedad crónica tienen un curso silencioso, prácticamente asintomático y, en consecuencia, la información publicada es heterogénea. 


\section{A León-Buitimea, A Leija-Salas, G Garrido-Fariña, J Reyes-Esparza, L Rodríguez-Fragoso}

Se ha descrito la velocidad de progresión del daño de la hepatitis $C$ en la etapa de infección aguda a la cirrosis en varios cohortes de pacientes seguidos en estudios prospectivos, y se ha demostrado que durante las primeras dos décadas se desarrolla cirrosis en aproximadamente $25 \%$ de los individuos. Los factores identificados en este subgrupo de enfermos son: la edad de contagio, el género masculino, la ingestión de alcohol y la infección concurrente por el virus de hepatitis B o el virus de inmunodeficiencia humana (VIH).

Una preocupación real se refiere al grupo de enfermos con cirrosis hepática secundaria al VHC y su pronóstico, ya que tienen una sobrevida muy baja. La cirrosis activa altera invariablemente la función hepática, evidenciada inicialmente por alteraciones serológicas y bioquímicas y, más tarde, por manifestaciones clínicas, tales como ictericia, ascitis, edema y, finalmente, la falla hepática que le ocasionará la muerte. La destrucción progresiva de los hepatocitos usualmente lleva esa secuencia de eventos. Sin embargo, en algunos casos, los cambios vasculares intrahepáticos, hemorragia de várices esofágicas o gástricas, infecciones intercurrentes, encefalopatía hepática o el desarrollo de carcinoma hepatocelular juegan un papel importante. Se considera que la muerte se presenta cuando más del $60 \%$ de los hepatocitos son destruidos. Ocasionalmente la cirrosis puede permanecer inactiva por largos periodos. Las lesiones inactivas, frecuentemente denominadas cirrosis latente, pueden no producir signos o síntomas de disfunción hepática. Algunas veces la enfermedad se complica por la presencia de hipertensión portal, desarrollo de várices hemorrágicas, ascitis e insuficiencia hepática y, en casos más severos, el desarrollo de carcinoma hepatocelular.

El curso de la enfermedad frecuentemente se puede complicar por la presencia de hipertensión portal. La muerte de la mayoría de los pacientes cirróticos es causada por el desarrollo de la hipertensión portal más que por el resultado de la función hepatocelular per se.
La fibrosis hepática y su etapa final, la cirrosis, representan un enorme problema de salud a nivel mundial, por el alto costo que implica el manejo y cuidado de los pacientes. Debido a lo anterior es imprescindible diseñar estrategias de manejo con el objetivo de modificar la historia natural de la enfermedad, aun en ausencia de la erradicación viral, pues la morbimortalidad asociada a la enfermedad es consecuencia directa de la fibrosis avanzada (cirrosis hepática).

\section{Modelos in vivo para la bioevaluación de fármacos con potencial antifibrótico.}

Los modelos animales son indispensables para el elucidar los mecanismos moleculares y celulares que llevan a la enfermedad y para el estudio de nuevos fármacos para el tratamiento de la fibrosis hepática. Por tanto, para evaluar el potencial antifibrótico de un agente determinado, es muy importante hacer una selección apropiada del modelo animal que reproduzca más estrechamente la enfermedad hepática crónica del humano. Desafortunadamente, la mayoría de los modelos de daño hepático que se han venido empleando para el estudio de diversos fármacos inducen la formación de radicales libres, necrosis severa e inflamación. La enfermedad hepática crónica del humano se caracteriza frecuentemente por moderada o incluso ausente inflamación y necrosis, a pesar de la progresión a la cirrosis. De ahí que muchos fármacos como los atrapadores de radicales libres, antioxidantes o anti-inflamatorios prevengan la fibrosis en esos modelos, pero sean ineficientes en el humano (37).

\section{Enfoques terapéuticos del tratamiento de la enfermedad hepática crónica.}

Los grandes avances en la comprensión de los mecanismos celulares y moleculares que participan en la fibrosis han facilitado el desarrollo de terapias efectivas más cercanas a la realidad. El fármaco ideal debe ser uno que sea fácilmente entregado y bien tolerado, con alta especificidad hepática y pocos efectos adversos. Esta terapia 


\section{Tratamiento de la enfermedad hepática crónica}

debe promover la resorción del exceso de matriz intersticial sin suprimir su papel fisiológico en el tejido hepático normal. El objetivo no es suprimir enteramente la fibrosis, sino más bien atenuar su desarrollo de tal manera que los pacientes con enfermedad hepática crónica no fallezcan por la falla orgánica final.

Las terapias antifibróticas empleadas en un inicio fueron enfocadas, en realidad, a eliminar la inflamación más que a suprimir la fibrosis. En la actualidad, tomando en consideración las bases moleculares y celulares de la fibrosis hepática, las estrategias terapéuticas están enfocadas, principalmente, hacia la regulación de las células estelares y los mediadores fibrogénicos. De ahí que, en la búsqueda de nuevas terapéuticas para el tratamiento de la enfermedad hepática crónica se incluyen esfuerzos para: remover el estímulo dañino, suprimir la inflamación hepática, controlar la activación de la célula estelar, y promover la degradación de la matriz extracelular.

Asimismo, con fundamento en el actual conocimiento de las bases fisiopatológicas de la fibrogénesis causada por virus de hepatitis $C$ en el hígado, los agentes antifibróticos pueden ser categorizados como sigue:

1) Agentes para erradicar el virus de hepatitis C.

2) Agentes para la citoproteción. Reducción de la inflamación y muerte celular.

3) Agentes para la inhibición de la activación de la célula estelar.

4) Agentes para la promoción de la degradación de la matriz.

5) Uso de terapia génica.

La mayoría de los agentes, en cada una de esas categorías, han sido estudiados en cultivos celulares y en varios modelos de fibrosis hepática en animales y se han propuesto para tratar a pacientes infectados con el virus de hepatitis C con fibrosis hepática. Sin embargo, su aplicación terapéutica en situaciones clínicas es, o bien, muy limitado, o de eficiencia incierta, debido a que los efectos secundarios severos (reacciones tóxicas) han dificultado su uso sistémico y continuo. Así que hay una sostenida necesidad de buscar y estudiar nuevos fármacos que sean más eficaces y que tengan menos reacciones adversas.

\section{Estrategias terapéuticas empleadas hasta el momento.}

La falta de métodos diagnósticos tempranos, que sean de bajo costo y aplicables a la población susceptible de desarrollar cirrosis hepática no permite diagnosticar los casos tempranos. De ahí que, por lo general, se atiende en la clínica a un número elevado de pacientes terminales con fibrosis muy avanzada. Por consiguiente, las estrategias que se deben plantear para tratar la fibrosis hepática deben de incluir medidas que permitan prevenir o bloquear los mecanismos que estimulan la síntesis de matriz, así como aquellas que incrementen su degradación. Este último punto es de gran importancia debido a que la mayoría de los pacientes que acuden a la clínica ya tienen fibrosis y no basta con prevenir la producción de colágena, sino que es necesario estimular la degradación del tejido fibroso cicatricial ya existente en el parénquima hepático (38).

A continuación describiremos brevemente algunos de los fármacos utilizados para el tratamiento de la fibrosis hepática tanto a nivel experimental como clínico, (ver cuadro 1) (39).

\section{1) Remoción del estímulo agresor.}

La remoción de la causa fundamental del daño hepático es la vía más efectiva para prevenir la fibrosis. Debido a la alta prevalencia del virus de hepatitis $\mathrm{C}$, hay esfuerzos masivos para erradicar el virus en pacientes infectados crónicamente.

Recientes estudios han demostrado que existe una clara mejoría histológica en pacientes responsivos a la terapia antiviral con interferón (IFN)/ribavirina para el virus de hepatitis C. Más allá de sus efectos antivirales, el IFN- $\alpha$ puede tener actividad antifibrótica directa, la cual puede explicar los reportes preliminares citando un 
A León-Buitimea, A Leija-Salas, G Garrido-Fariña, J Reyes-Esparza, L Rodríguez-Fragoso

\section{Cuadro 1}

Blancos terapéuticos en la fibrosis hepática.

\begin{tabular}{|ll|}
\hline \multicolumn{2}{|c|}{ Fibrosis en progreso o ya establecida } \\
\hline Reducción de la inflamación & \\
\hline $\begin{array}{l}\text { Remoción del agente dañino (Agentes antivirales: } \\
\text { IFN/ribavirina y lamivudine). }\end{array}$ & Colchicina \\
TNF- $\alpha$ & Antagonistas del receptor de la IL-10 \\
Octreotide & Corticoides. \\
Malotilato & \\
\hline Inhibición de la activación de las células estelares & Resveratrol \\
\hline Citoquinas: IFN's, HGF, etc. & Quercetin \\
Antioxidantes: Vitamina E. & N-acetilcisteína \\
Salimarina & Pentoxifilina \\
\hline Inhibición de la perpetuación de la activación de células estelares (Antiproliferativos) \\
\hline Transilast & Antagonistas del TGF- $\beta 1$ \\
\hline Inhibición de la secreción de matriz rica en colágena por células estelares (Antifibrogénicos) \\
\hline HGF & Transilast \\
Antagonistas de los receptores de la endotelina & \\
\hline Silimarina & \\
\hline \multicolumn{1}{|l|}{ Incremento o inicio de la resolución de la fibrosis. } \\
\hline Estimulación de la apoptosis en las células estelares. \\
\hline $\begin{array}{ll}\text { Gliotoxina. } \\
\text { NGF }\end{array}$ \\
\hline Degradación de la matriz rica en colágena. & Antagonistas de las integrinas. \\
\hline Relaxina. & \\
Antagonistas del TIMP 1 y 2 & \\
\hline Modificado de Bataller y Brenner, 2001 (38). & \\
\hline
\end{tabular}

efecto antifibrótico de IFN/ribavirina, incluso en pacientes que fallan para depurar el virus.

Hay tres isoformas de interferones (IFN), $\alpha, \beta$ y $\gamma(40)$, cada una de ellos tienen diferentes acciones biológicas. Hay múltiples subtipos de IFN- $\alpha$, mientras que sólo hay una especie de IFN- $\beta$ e IFN$\gamma$. IFN- $\alpha$ y $\beta$ se unen al mismo receptor y están muy estrechamente relacionados tanto estructural como funcionalmente. El IFN- $\alpha$ es estimulado por la infección viral y el IFN- $\gamma$ es estimulado por estímulos mitogénicos y antigénicos de linfocitos $\mathrm{T}$ o células asesinas. El IFN- $\alpha$ tiene muchos más efectos antivirales que el IFN- $\gamma$; mientras que IFN- $\gamma$ es 100 a 10,000 veces más potente como inmunoestimulador que el IFN- $\alpha$ (41). Esta Revista Biomédica observación ha conducido al concepto que los IFN$\alpha$ y $\beta$ son primariamente agentes antivirales con algunos efectos inmunomoduladores, pero el IFN$\gamma$ es primariamente un agente inmunomodulador con poco efecto antiviral (42).

Varios reportes clínicos recientes han llevado a la conclusión de que el tratamiento con IFN- $\alpha$ es efectivo para reducir y eliminar el ARN sérico del VHC $(43,44)$ mejorando con ello el grado de fibrosis hepática y la necroinflamación (4548). Los IFNs también han mostrado reducir la expresión de $\alpha$-actina de músculo liso (SMA) en el hígado (49), lo cual se asocia con una disminución de la colágena in vitro, y con una disminución de los niveles de ARNm de procolágena tipo I, III y 
Tratamiento de la enfermedad hepática crónica

IV $(50,51)$. Aunque el modo de acción de los IFN (directo o indirecto) sobre la fibrosis hepática no es absolutamente claro hasta el presente, ha sido reportado que los niveles de ARNm de TGF- $\beta 1$, así como de procolágena tipo I están disminuidos significativamente en pacientes respondedores a la terapia con IFN (52). Además, ha sido señalado que el IFN- $\alpha$ puede inhibir la proliferación de células estelares hepáticas en cultivos primarios (40). Estos hallazgos sugieren que el efecto primario del IFN- $\alpha$ es inhibir el daño inducido por el VHC.

La ribavirina es un análogo sintético de guanosina que ejerce actividad anti-viral in vitro contra un rango de virus ADN y ARN. Tiene un efecto sinergista cuando se usa junto con IFN- $\alpha$. Sin embargo, la ribavirina no es recomendada como monoterapia debido a que no tiene efecto sobre la depuración sérica del ARN del VHC. La administración de IFN- $\alpha$ y ribavirina, conjuntamente, produce una respuesta de depuración viral superior que el IFN solo (53, 54). Se ha reportado que la terapia combinada de IFN- $\alpha$ y ribavirina reducen significativamente la progresión de la fibrosis en pacientes con VHC (55).

En contraste con el IFN- $\alpha$, el IFN- $\gamma$ es inefectivo para erradicar el ARN sérico del VHC, pero hallazgos experimentales sugieren que el IFN$\gamma$ puede inhibir la activación de la célula estelar (40, 41) y, como consecuencia, reduce grandemente la fibrosis hepática $(56,57)$. En relación al uso de IFN- $\gamma$ en pacientes con infección por VHC, hay poco interés clínico debido a que la sobre-expresión de IFN- $\gamma$ en hígado lleva a la hepatitis crónica y además por el potencial de efectos adversos que produce a largo plazo, relacionado con sus efectos inmunomoduladores.

\section{2) Supresión de la inflamación hepática.}

Los mediadores de la inflamación pueden estimular la activación de la célula estelar en la enfermedad hepática crónica. De manera que los fármacos anti-inflamatorios pueden ser benéficos para prevenir la inflamación en esas condiciones.
Los corticoides han sido usados para el tratamiento de muchas enfermedades inflamatorias del hígado (58). Sin embargo, la incompleta supresión de la fibrogénesis y los considerables efectos secundarios indeseables después de uso prolongado, limitan su uso.

La colchicina es un fármaco anti-inflamatorio, pero su valor en el tratamiento de la enfermedad hepática crónica es controversial. Desde hace años este fármaco se ha utilizado para el tratamiento de las enfermedades hepáticas basándose en su capacidad antiinflamatoria, antimitótica y, fundamentalmente, antifibrogénica. De hecho, la colchicina impide el transporte microtubular del colágeno y aumenta la actividad colagenolítica (5962). Diferentes ensayos clínicos aleatorizados han evaluado la eficacia de este fármaco en pacientes con fibrosis y cirrosis alcohólica y no alcohólica. La mayoría de los estudios han aportado resultados poco alentadores, pero en un estudio, publicado en 1988 y realizado en México, se observó que el tratamiento con colchicina se asociaba con una mayor sobrevida de los pacientes cirróticos de causa diversa. Un estudio multicéntrico de 14 ensayos clínicos aleatorizados, que incluyeron 1138 pacientes, indicó que el tratamiento con colchicina no se asoció con una menor mortalidad global, ni con mortalidad de causa hepática. Sin embargo, el uso de la colchicina se asoció con una elevada incidencia de efectos adversos. En el momento actual no parece, por tanto, existir ninguna evidencia científica para prescribir este fármaco en la fibrosis hepática. No obstante esta ambigüedad, la colchicina está siendo aún utilizada por algunos médicos y estudios recientes sugieren que su metabolito, puede ser el que tiene la actividad antifibrogénica (63-65).

El malotilato es un agente anti-inflamatorio experimental el cual tiene efectos hepatoprotectores y reduce la fibrogénesis experimental hepática inducida con $\mathrm{CCl}_{4}$ o dimetilnitrosamida. Su mecanismo de acción se cree que lo ejerce a través de suprimir la expresión del citocromo $\mathrm{P}_{450}$. Sin embargo, no hay beneficios claros demostrados 


\section{A León-Buitimea, A Leija-Salas, G Garrido-Fariña, J Reyes-Esparza, L Rodríguez-Fragoso}

en ensayos clínicos (66,67).

El transilast, un agente anti-alérgico, también ha mostrado reducir la proliferación y la síntesis de la colágena en células de músculo liso vascular (68). Asimismo, inhibe la activación y expresión de TGF- $\beta 1$ en células estelares hepáticas cultivadas in vitro (69).

Otra estrategia anti-inflamatoria es neutralizar las citoquinas inflamatorias usando antagonistas de receptores específicos. El antagonista del receptor para Interleucina-1 da un modesto efecto en ratas con daño hepático inducido con dimetilnitrosamina (70). Un análogo sintético de las secuencias de aminoácidos Arg-Gly-Asp (RGD), el cual representa una molécula de unión a integrinas activado por fibronectina y otras moléculas de la matriz extracelular, ha sido usado en el daño hepático inducido con concanavalina en ratones (71). En el mismo estudio, el pretratamiento de los animales con el receptor de TNF- $\alpha$ redujo efectivamente la elevación de enzimas hepáticas y bloqueó la liberación de TNF- $\alpha$ e IL-6. La reducción de los niveles de citoquinas fue acompañada por disminución de la necrosis e inflamación en secciones tisulares.

Octreotide, un análogo sintético de la somatostatina, se ha estudiado recientemente en modelos de fibrosis hepática inducida por ligadura de conducto biliar en la rata. El compuesto redujo el flujo portocolateral en esos modelos y también mostró un efecto antifibrótico en el daño hepático inducido por el $\mathrm{CCl}_{4}$. La actividad antifibrótica del octreotide puede ser atribuida a sus propiedades anti-inflamatorias (72).

\section{3) Regulación de la activación de la célula estelar.}

La reducción del estrés oxidativo, el cual es un importante estímulo para la activación es también una estrategia terapéutica empleada. Los antioxidantes, como la vitamina E, suprimen la fibrogénesis en algunos (73) pero no en todos los modelos de fibrosis experimental. Estudios recientes han documentado la inhibición de la célula estelar por resveratrol, quercetin y Nacetilcisteína (74).

La silimarina es un compuesto de origen natural que ha exhibido propiedades antifibróticas prometedoras en el daño hepático experimental. De acuerdo a su estructura, la silimarina pertenece a un grupo de compuestos flavonoides, junto con quercetin, baicalina y baicaleina. Esos flavonoides han atraído también la atención debido a sus propiedades antifibrogénicas $(75,76)$. La silimarina reduce la acumulación de colágena en un $30 \%$ en la fibrosis biliar inducida en la rata (77). Funciona como un antioxidante y puede disminuir el daño hepático tanto por citoprotección como por inhibición de la función de las células de Kupffer $(78,79)$. Un ensayo en humanos ha reportado un ligero incremento en la sobrevivencia en cirróticos alcohólicos comparado con controles no tratados (75).

La modulación de la actividad de citoquinas representa una aproximación relativamente fácil y específica. La terapia incluye antagonistas de receptores y anticuerpos contra citoquinas, inhibición de su producción o activación y uso de citoquinas o proteínas las cuales promueven la resorción de la matriz (80).

Los antagonistas del factor de crecimiento transformante beta (TGF- $\beta$ ) están bajo intensa investigación. Al neutralizarse esta importante citoquina fibrogénica se puede en gran medida regular a la baja ("down-regulation") la producción de matriz (8)). Varios antagonistas de TGF- $\beta$ están siendo desarrollados y evaluados, incluyendo al receptor soluble del TGF- $\beta$ tipo II y los nucleótidos anti-sentido. Por ejemplo, el receptor del TGF- $\beta$ tipo II inhibe la activación de la célula estelar y la fibrogénesis in vivo cuando se administró ya sea antes o después del estímulo fibrogénico $(82,83)$. Otras estrategias para bloquear funcionalmente el TGF- $\beta$ están bajo estudio, incluidas las proteínas secuestradoras de TGF- $\beta$, como el decorin o el péptido asociado a latencia $(84,85)$.

Los antagonistas del receptor de endotelina también han sido probados como agentes

\section{Revista Biomédica}




\section{Tratamiento de la enfermedad hepática crónica}

antifibrogénicos y son los más prometedores, porque agentes de este tipo están bajo ensayos clínicos para enfermedades hipertensivas, Bosentan, un antagonista del receptor de endotelina, es antifibrótico y reduce la activación de la célula estelar en la fibrosis experimental (86).

El factor de crecimiento del hepatocito (HGF) inhibe la fibrosis hepática y promueve la regeneración hepática en modelos animales de daño hepático $(87,88)$. Una variante del HGF es efectiva para inhibir la activación de las células estelares hepáticas, regular a la baja la expresión del ARNm de pro-colágenas y TGF- $\beta 1$, y estimular la regeneración hepática (89).

La relaxina es un péptido que disminuye la síntesis de colágena e incrementa la degradación de la matriz in vivo e in vitro $(90,91)$. A la fecha este agente no se ha usado en modelos de fibrosis hepática, aunque su eficacia ha sido reportada en otros tejidos, incluido el pulmón.

La pentoxifilina, un análogo de la metilxantina, es clínicamente útil para el tratamiento de estados clínicos involucrando defectos en la microcirculación regional. Fue empleado para inhibir la transición de la célula estelar hepática a células semejantes a miofibroblastos (CEH activada) y para inhibir la proliferación de la células in vitro (92). Otro estudio mostró que la pentoxifilina inhibió la producción de TNF$\alpha$ estimulada por LPS en monocitos así como también bloqueó la activación del factor nuclear $\kappa \mathrm{B}(\mathrm{NF}-\kappa \mathrm{B})$ en célula estelar hepática in vitro e in vivo (aisladas de ratas tratadas con $\mathrm{CCl}_{4}$ ) (93). También redujo la toxicidad hepática aguda del $\mathrm{CCl}_{4}$ (94). Fue recientemente reportado que la pentoxifilina reduce la proliferación y activación de las células estelares (expresión de alfa 1 actina de músculo liso) en modelos animales de fibrosis hepática inducida por $\mathrm{CCl}_{4} \mathrm{y}$ ligadura del conducto biliar (95).

El factor de crecimiento derivado de plaquetas (PDGF) es el más potente mitógeno para las células estelares hepáticas in vitro, y probablemente también juega un importante papel en la transformación de la célula estelar a células semejantes a miofibroblastos in vivo (96). La activación del receptor para PDGF por autofosforilación de su residuo tirosina es un evento crucial en la transducción de señales, corriente abajo. El acoplamiento del ligando, PDGF-aa, ab, o bb al correspondiente dímero del receptor causa autofosforilación de la tirosina en la subunidad del receptor, el cual inicia los eventos corriente abajo, como la mitogenesis y la oscilación del calcio en la célula estelar (97). El PD 98059 es un inhibidor específico de la activación de la señal activada por cinasas (ERK) en la célula estelar para su proliferación (98). Esos experimentos han mostrado que el bloquear la vía de transducción de señales puede ser una alternativa para interrumpir el papel de las citoquinas en la célula estelar y para minimizar la fibrogénesis.

\section{4) Promover la degradación de la matriz extracelular.}

Esta estrategia es de especial significado clínico dada la necesidad de reducir la matriz en pacientes con fibrosis establecida. En la actualidad esta estrategia terapéutica está siendo ampliamente explorada. Por ejemplo, prevenir la sobrerregulación de los inhibidores de metaloproteasas tisulares 1 y 2 (TIMP 1 y 2 ) durante la activación de la célula estelar puede incrementar la degradación de la matriz extracelular in vivo (99). Estrategias para incrementar la actividad de las enzimas que degradan las proteínas de la matriz son también estudiadas. Antagonistas del TGF- $\beta$ pueden estimular la degradación de la matriz tanto por regular a la baja los inhibidores tisulares de metaloproteasas como por incrementar la actividad neta de colagenasa intersticial. La relaxina puede directamente incrementar la degradación de la matriz (100). El uso de promotores de la apoptosis de la célula estelar es otra estrategia potencial, pero es aún poco factible en la práctica.

No obstante la existencia de las estrategias empleadas para aliviar las enfermedades hepáticas 


\section{A León-Buitimea, A Leija-Salas, G Garrido-Fariña, J Reyes-Esparza, L Rodríguez-Fragoso}

crónicas experimentales, resulta más evidente que la estrategia ideal para el tratamiento de la enfermedad hepática crónica debe incluir la prevención de la fibrogénesis, la inducción de la proliferación de las células hepáticas, la formación de nuevos vasos y la reorganización de la arquitectura hepática. Como hemos visto a lo largo de esta revisión, un gran número de nuevos fármacos han surgido, los cuales han mostrado que tienen importantes efectos sobre el metabolismo de la célula estelar y que influyen fuertemente en su conducta in vivo. Sin embargo, aunque muchos de ellos han sido evaluados tanto en modelos experimentales in vivo e in vitro, pocos de ellos han probado ser realmente efectivos en el tratamiento de la fibrosis y cirrosis en el humano.

\section{5) Aplicación de la terapia génica.}

La terapia génica es un nuevo modo de tratamiento para enfermedades hereditarias y adquiridas basado en la transferencia de material genético a los tejidos. Los genes son incorporados dentro de vectores apropiados para facilitar su entrada y función dentro de las células blanco, los vectores virales son frecuentemente usados debido a su alta eficiencia de transducción. Un considerable número de estudios preclínicos indican que una gran variedad de enfermedades hepáticas incluidas la fibrosis y cirrosis hepática puede ser tratadas a través de la terapia génica. Las enfermedades hepáticas son un reflejo de los éxitos y fracasos de la terapia génica en general $(101,102)$

En el tratamiento de las enfermedades por los virus de hepatitis B y C la terapia génica ha sido usada para mejorar la actividad regional de distintas citoquinas, incluyendo el interferón alfa, el interferón gamma, la interleucina 10 ó la interleucina 12, permitiendo reducir sus efectos adversos a nivel sistémico (103-105). Esta estrategia podría ser especialmente útil para pacientes que recaen tras una respuesta inicial a los tratamientos disponibles. Por otro lado, la transferencia génica puede hacer que las células sean resistentes a la infección viral bloqueando el ciclo vital del virus en distintos puntos. La interferencia con la propagación del virus podría lograrse utilizando moléculas antisentido, ribosomas o ARN interferentes, pero ninguna de estas estrategias ha pasado a la fase de ensayos clínicos (106, 107).

La terapia génica en la fibrosis hepática está enfocada fundamentalmente a la prevención de la fibrogénesis y a la estimulación de la regeneración hepática. El TGF- $\beta$ juega un papel importante en la fibrogénesis y su acción puede modularse de varias formas. Por un lado, se puede bloquear específicamente su efecto haciendo que las células hepáticas expresen en su membrana una forma alterada del receptor (108). O bien, se puede disminuir su acción. En ambos casos la disminución de la acción de la actividad del TGF$\beta$ tiene como resultado una menor fibrosis en los modelos animales. Por otro lado, la producción tanto a nivel sistémico como local de agentes favorecedores de la regeneración como el factor de crecimiento de hepatocitos (HGF) o el factor semejante a la insulina (IGF-1) reduce la fibrosis y aumenta la sobrevivencia de los animales cirróticos (109)

\section{Conclusiones y direcciones futuras.}

El objetivo final de la investigación en la hepatofibrogénesis es desarrollar las bases racionales para una terapia antifibrótica efectiva. Aunque los avances en la comprensión de los mecanismos mediados por el virus de hepatitis C han llevado al desarrollo de nuevas terapias efectivas en la práctica clínica, no hay terapias definidas y establecidas para la fibrosis hepática. Sin embargo, recientes hallazgos en la patogénesis molecular de la fibrosis hepática y el papel de la célula estelar activada proporcionan esperanzas para el desarrollo futuro de terapias exitosas. Las expectativas sobre la terapia génica se han exagerado y en ocasiones han impedido ver que en realidad continúa siendo una técnica experimental y no una terapia clínica establecida

\section{Revista Biomédica}




\section{Tratamiento de la enfermedad hepática crónica}

y eficaz. Los obstáculos que impiden el éxito clínico de la terapia génica tienen que ver más con las herramientas que con sus fundamentos. En general, los factores necesarios para conseguir que la terapia génica sea efectiva no son diferentes de los de otras modalidades terapéuticas nuevas. Se incluyen los factores técnicos (la distribución y expresión genética), clínicos (eficacia y seguridad terapéutica) y factores socioeconómicos.

\section{AGRADECIMIENTOS.}

A Luz María Herrera Recamier, por su amable revisión del manuscrito.

\section{REFERENCIAS.}

1. Alter MJ, Kruszon-Moran D, Nainan OV, McQuillan GM, Gao F, Moyer LA. The prevalence of hepatitis C virus infection in the United States, 1998 through 1994. N Eng J Med 1999; 341:556-62.

2. Liang TJ, Rehermann B, Sep LB, Hoofnagle JH. NIH Conference, Pathogenesis, natural history, treatment and prevention of hepatitis C. Ann Intern Med 2000; 132:296305.

3. Méndez-Sánchez N, Baptista-González H, SánchezGómez RH, Bordes-Aznar J, Uribe M. The prevalence of hepatitis B and C in blood donors in a 3rd level hospital of Mexico City. Salud Publ Mex 1999; 41:475-8.

4. Uribe M, Méndez-Sánchez N. Consenso nacional sobre hepatitis C. Rev Gastroenterol Mex 2002; 67:S7-8.

5. National Institutes of Health Consensus Development Conference Statement: Management of Hepatitis C: 2002. Hepatology 2002; 36:S3-15.

6. El-Serag HB, Mason AC, Rising incidence of hepatocellular carcinoma in the United States. N Engl J Med 1999; 340:745-50.

7. Wong JB, McQuilan GM, McHutchinson JG, Poynard T. Estimating future hepatitis $C$ morbidity, mortality and cost in the United States. Am J Public Health 2000; 90:1562-9.

8. Valkova M. Hepatic fibrogenesis. Bratisl Lek Lis 2002; 103:76-85.

9. $\quad$ Eng FJ, Friedman SL. Fibrogenesis. Am J Physiol Gastroenterol Liver Physiol 2000; 279:G7-11.
10. Arthur MJ. Matrix degradation in liver: a role in injury and repair. Hepatology 1997; 26:1069-71.

11. Gressner AM. Mediators of hepatic fibrogenesis. Hepatogastroenterology 1996; 43:92-103.

12. Tsukamoto H. Cytokine regulation of hepatic stellate cells in liver fibrosis. Alcoholism Clin Exp Res 1999; 23: 911-6.

13. Friedman SL. Molecular regulation of hepatic fibrosis, an integrated cellular response to tissue injury. J Biol Chem 2000; 275:2247-50.

14. Schuppan D. Structure of extracellular matrix in normal and fibrotic liver: collagens and glycoproteins. Sem Liver Dis 1990; 10:1-10.

15. Schuppan D, Gressner AM. Functions and metabolism of collagens and other extracellular matrix proteins. In: Bircher J, Benhamon JP, Rodes J, (Editores). Oxford Textbook of Clinical Hepatology, Oxforf University Press 1999. p. 381-406.

16. Milani S, Herbst H, Schuppan D, Hahn EG, Stein H. In situ hybridization for procollagen types I, III and IV mRNA in normal and fibrotic rat liver: evidence for predominant expression in nonparenquimal liver cells. Hepatology 1989; 10:84-92.

17. Neubauer K, Saile B, Ramadori G. Liver fibrosis and altered matrix synthesis. Can J Gastroenterol 2001; 15:18793.

18. Sato S, Leo MA, Lieber CS. Ultrastructural localization of type III procollagen in baboon liver. Am J Pathol 1986; 122:212-7.

19. Friedman SL. The cellular basis of hepatic fibrosis. N Engl J med 1993; 328:1826-35.

20. Brenner DA, Waterboer T, Choi SK. New aspects of hepatic fibrosis. J Hepatol 2000; 32:32-8.

21. Gressner AM, Bachem MG. Molecular mechanisms of liver fibrogenesis - A homage to the role of activated fat storing cells. Digestion 1995; 56:335-46.

22. Friedman SL. Liver fibrosis - from bench to bedside. J Hepatol 2003; 38:S38-53. 


\section{A León-Buitimea, A Leija-Salas, G Garrido-Fariña, J Reyes-Esparza, L Rodríguez-Fragoso}

23. Rai RM, Loffreda S, Karp CL, Yang SQ, Lin HZ, Diehl AM. Kupffer cells depletion abolishes induction of interleukin-10 and permits sustained overexpression of tumor necrosis factor alpha messenger RNA in the regeneration rat liver. Hepatology 1997; 25:889-95.

24. Hellerbrand C, Stefanoc B, Giordano F, Burchardt ER, Brenner DA. The role of TGF $\beta 1$ in initiating hepatic stellate cell activation in vivo. J hepatol 1999; 30:77-87.

25. Dooley S, Delvoux B, Lahme B, Mangasser-Stephan K, Gressner AM. Modulation of transforming growth factor beta response and signaling during transdifferentiation of rat hepatic stellate cell to myofibroblasts. Hepatology 2000; 31: 1094-106.

26. Mallat A, Gallois C, Tao J, Habib A, Maclouf J, Mavier P, Preaux AM, Lotersztajn S. Platelet-derived growth factor-BB and trombin generate positive and negative signals for human hepatic stellate cell proliferation. Role of prostaglandin/cyclic AMP pathway and cross-talk with endothelin receptors. J Biol Chem 1998; 273:27300-5.

27. Olaso E, Friedman SL. Molecular regulation of hepatic fibrosis. J Hepatol 1998; 29:836-42.

28. Friedman SL. The virtuosity of hepatic stellate cells. Gastroenterol 1999; 117:1244-6.

29. Hoffman C, Lahne B, Brenzel A, Gressner AM. The relation between hepatocellular damage and activation of fat storing cells proliferation in vitro. Inter Hepatol Commun 1994; 2:29-36.

30. Friedman SL. Molecular regulation of hepatic fibrosis, an integrated cellular response to tissue injury. J Biol Chem 2000; 275:2247-50

31. Gressner AM. Cytokines and cellular crosstalk involved in the activation of fat-storing cells. J Hepatol 1995; 22:S28-36.

32. Shimizu I. Antifibrogenic therapies in chronic $\mathrm{HCV}$ infection. Curr Drug Targets Infect Disord. 2001; 1:22740.

33. Yin M, Wheeler MD, Kono H, Bradford B, Gallucci RM, Luster MI, Thurman RG. Essential role of tumor necrosis factor alpha in alcoholic-induced liver injury in mice. Gastrenterol 1999; 117:942-52.

34. Pinzani M. Hepatic stellate (Ito) cells: expanding roles for a liver specific pericyte. J Hepatol 1995; 22:700-6.
35. Rosenbaum J, Blazejewski S. Regulation of Ito cell proliferation by soluble factors. J Hepatol 1995; 22:65-70.

36. Rockey DC. The cell and molecular biology of hepatic fibrogenesis. Clinical and therapeutics implications. Clin Liver Dis 2000; 4:319-55.

37. Schuppan D, Strobel D, Hahn EG. Hepatic fibrosisTherapeutics strategies. Digestion 1998; 59:385-90.

38. Bataller R, Brenner DA. Hepatic stellate cells as a target for the treatment of liver fibrosis. Semin Liver Dis 2001; 21:437-51.

39. Safadi R, Friedman SL. Hepatic fibrosis - Role of hepatic stellate cell activation. Med Gen Med 2002; 15; 4: 27.

40. Mallat A, Preaux AM, Blazejewski S. Interferon alpha and gamma inhibit proliferation and collagen synthesis of human ito cells in culture. Hepatology 1995; 21:1003-10.

41. Lu G, Shimizu I, Cui X, Itonaga M, Tamaki K, Fukuno $\mathrm{H}$, Inoue $\mathrm{H}$, Honda $\mathrm{H}$, Ito $\mathrm{S}$. Interferon alpha enhances biological defense activities against oxidative stress in cultured rat hepatocytes and hepatic stellate cells. J Med Invest 2002; 49:172-81.

42. Rockey DC, Maher JJ, Jarnagin WR, Gabbiani G, Friedman SL. Inhibition of rat lipocyte activation in culture by interferon gamma. Hepatology 1992; 16:776-84.

43. Abe S, Tabaru A, Ono M, Tai M, Narita R, Moriyama A, Otsuki M. High dose interferon-alpha therapy lowers the levels of serum fibrogenesis markers over 5 years in chronic hepatitis C. Hepatol Res 2003; 25:22-31.

44. Manabe N, Chevallier M, Chossegros P. Interferon alpha therapy reduces liver fibrosis in chronic non $\mathrm{A}$, non $\mathrm{B}$ hepatitis; a quantitative histological evaluation. Hepatology 1993; 18:1344-49.

45. Camma C, Giunta M, Linea C. The effect of interferon on the liver in chronic hepatitis C; a quantitative evaluation of histology by meta-analysis. J Hepatol 1997; 26:118799.

46. Rosenbloom J, Feldman G, Freundhich G, Jiménez SA. Transcriptional control of human diploid fibroblast collagen synthesis by interferon. Biochem Biophys Res Commun 1984; 123:365-72.

\section{Revista Biomédica}




\section{Tratamiento de la enfermedad hepática crónica}

47. Suou T, Hosho K, Kishimoto Y. Long-term decrease in serum $\mathrm{N}$-terminal propeptide of type III procollagen in patients with chronic hepatitis $\mathrm{C}$ treated with interferon alpha. Hepatology 1995; 22:426-31.

48. Guido M, Rugge M, Chemello L. Liver stellate cells in chronic viral hepatitis; the effect of interferon therapy. J Hepatol 1996; 24:301-7.

49. Kirk AP, Jain S, Pocok S, Thomas HC, Sherlock S. Late results of the Royal Free Hospital prospective controlled trial of prednisone therapy in hepatitis B surface antigen negative chronic active hepatitis. Gut 1980; 21:78-83.

50. Bodenheimer H, Shaffner F, Pezzullo J, Evaluation of colchicine therapy in primary biliary cirrhosis. Gastroenterology 1988; 95:124-9.

51. Kaplan MM, Alling DW, Zimmerman HJ. A prospective trial of colchicine for primary biliary cirrhosis. N Engl J Med 1986; 315:1448-54.

52. Castilla A, Prieta J, Fausto N. Transforming growth factors beta-1 and alpha in chronic liver disease: effect of interferon-alfa therapy. N Engl J Med 1991; 324:933-40.

53. Poynard T, McHutchinson J, Davis GL. Impact of interferon alpha $2 \mathrm{~b}$ and ribavirin on progression of liver fibrosis in patients with chronic hepatitis C. Hepatology 200; 32:1187-99.

54. Poynard T, McHutchinson J, Manns M, Trepo C, Linsay K, Goodman Z, Ling M, Albrecht J. Impact of pegylated interferon alpha $2 \mathrm{~b}$ and ribavirin on liver fibrosis in patients with chronic hepatitis C. Gastroenterology 2002; 122:1303-13.

55. Poynard T, Moussali J, Ratziu V. Effect of interferon therapy in "non responder" patients with chronic hepatitis C, EASL International Consensus Conference on Hepatitis C. J Hepatol 1999; 31 (Suppl 1):178-83.

56. Di Bisceglie AM, Thompson J, Smith-Wilkaitis N. Combination of interferon and ribavirin in chronic hepatitis C: re-treatment of nonresponder to interferon. Hepatology 2001; 33:704-7.

57. Rodríguez Fragoso L., Alvarez R., Reyes Esparza J.A., Garcés A. M.E. Acetaldehyde increases the activity and gene expression of plasminogen activator activity in a hepatic stellate cell line. Toxicology 1999, 137:1-11.
58. Bechade D, Oui B, Mayet F, Trouette H, Schouler L, Jouglen J, Raymond JM, Couzigou P. Appearance of hepatitis $\mathrm{C}$ virus replication and increase of aminotransferase activity after corticoid treatment of presumed autoimmune hepatitis. 2 cases. Gastroenterol Clin Biol 1996; 20:696-9.

59. Angelico M, Cepparulo M, Barlattani A, Liuti A, Gentile S, Hurtova M, Ombres D, Guarascio P, Rocchi $\mathrm{G}$, Angelico F. Unfavourable effects of colchicine in combination with interferon-alpha in the treatment of chronic hepatitis. Aliment Pharmacol Ther 2000; 14:1459-67.

60. Floreani A, Colloredo G, Lobello S, Di Marco M, Popovic A, Naccarato R. Preliminary results of a two-center trial with colchicine for the treatment of chronic hepatitis B. Am J Gastroenterol 2001; 96:3451-2.

61. Rodríguez L, Cerbón J, Muñoz-Moreno ML. Effects of colchicine and colchiceine in a biochemical model of liver injury and fibrosis. Archives of the Medical Research 1998, 29:109-17.

62. Kershenobich D, Vargas F, García-Tsao G, Tamayo RP, Gent M, Rojkind M. Colchicine in the treatment of cirrhosis of the liver. N Engl J Med 1988; 318:1709-13.

63. Mourelle M, Fraginals R, Rodríguez L, Favari L, Pérez-Alvarez V. Protective effect of colchiceine against acute liver damage. Life Science 1988; 45:891-900.

64. Rodríguez L, Mourelle M, Pérez V, Cerbón AJ. Reduction of hepatic collagen synthesis by colchiceine in cirrhotic rat liver. Biochem Pharmacol. 1990; 9:157-63.

65. Mourelle M, Favari L, Rodríguez L, Pérez V, Fraginals R. Colchiceine treatment prevents $\mathrm{CCl} 4$ induced rat liver cirrhosis. Biochem Pharmacol 1990; 9:165-9.

66. Ala-Kokko L, Stenback F, Ryhannen L. Preventive effect of malotilate on dimethynitrosamine-induced liver fibrosis in the rat. J Lab Clin Med 1989; 113:177-83.

67. Ala-Kokko L, Stenback F, Ryhannen L. Preventive effect of malotilate on carbon tetracloride induced liver damage and collagen accumulation in the rat. Biochem J. 1987; 246:503-9.

68. Tanaka K, Honda M, Kuramochi T, Morioka S. Prominent inhibitory effects of transilast on migration and proliferation of collagen synthesis by vascular smooth muscle cells. Atherosclerosis 1994; 107:322-7. 


\section{A León-Buitimea, A Leija-Salas, G Garrido-Fariña, J Reyes-Esparza, L Rodríguez-Fragoso}

69. Ikeda H,Inao M, Fujiwara K. Inhibitory effect of transilast on activation and trasforming growth factor beta 1 expression in cultured rat stellate cells. Biochem Biophys Res Commun 1996; 227:322-7.

70. Mancini R, Benedetti A, Jezequel AM. An interleukin1 receptor antagonist decreases fibrosis induced by dimethylnitrosamine in rat liver. Virchows Arch 1994; 424: 25-31.

71. Forrt J, Oberti F, Pilette C. Antifibrotic and hemodynamic effect of the early and chronic administration of octreotide in two models of liver fibrosis in rats. Hepatology 1998; 28:1525-31.

72. Karalis K, Mastorakos G, Chtousos GP, Tolis G. Somatostatin analogues suppress the inflammatory reaction in vivo. J Clin Invest 1994; 93:2000-6.

73. Brown KE, Poulos JE, Li L. Effect of vitamin E suplementation on hepatic fibrogenesis in chronic dietary iron overload. Am J Physiol 1997; 272:G116-123.

74. Kawada N, Seki S, Inoue M, Kuroki T. Effect of antioxidants, resveretrol, quercetin and $\mathrm{N}$-acetylcysteine, on the functions of cultured rat hepatic stellate cells and Kupffer cells. Hepatology 1998; 27:1265-74.

75. Ferenci P, Dragosics B, Dittrich H. Randomizedd controlled trials of sylimarin treatment in patients with cirrhosis of the liver. J Hepatol 1989; 9:105-10.

76. Geerts A, Rogiers V, Sho-saiko-to. The right blend of traditional oriental medicine and liver cell biology. Hepatology 1999; 29:282-4.

77. Boigk G, Stroedter L, Herbst H, Waldschmith J, Riecken EO. Sylimarin retards collagen accumulation in early and advanced biliary fibrosis secondary to complete bile duct obliteration in rats. Hepatology 1997; 26:643-9.

78. Dehmlow C, Erhard J, De Groot H. Inhibition of Kupffer cells functions as an explanation for the hepatoprotective properties of silibinin. Hepatology 1996; 23:749-54.

79. Pietrangelo A, Borella F, Casalgrandi G. Antioxidant activity of silybin in vivo during long-term iron overload in rats. Gastroenterology 1995; 109:1941-9.

80. Friedman SL, Cytoquines and fibrogenesis. Semin Liver Dis 1999; 19:129-40.

\section{Revista Biomédica}

81. Qi Z, Atsuchi N, Ooshima A, Takeshita A, Ueno H Blockade of type beta transforming growth factor signaling prevents live fibrosis and dysfunction in the rat. Proc Natl Acad Sci USA 1999; 96:2345-9.

82. Tsanf ML, Zhou L, Zheng BL. Characterization of recombinant soluble human transforming growth factorbeta receptor type II (rhTGF-beta sRII). Cytokine 1995; 7: 389-97.

83. Spearman M, Taylor WWR, Greenber AH, Wright JA. Antisense oligodeoxyribonucleotide inhibition of TGF$\beta 1$ gene expression and alteration in growth and malignant properties of mouse fibrosarcoma cells. Gene 1994; 149: 25-9.

84. Border WA, Noble NA, Yamamoto T. Natural inhibitor of transforming growth factor beta protects against scarring in experimental kidney disease. Nature 1992; 360:361-4.

85. Roth S. Michel K, Gressner AM. (Latent) Transforming growth factor beta in liverparenchimal cells, its injurydependent release, and paracrine effects on rat hepatic stellate cells. Hepatology 1998; 27:1003-12.

86. Luscher TF, Wenzel RR. Endothelin and endhotelin antagonists: pharmacology and clinical implications. Agents Actions 1995; 45:237-53.

87. Matsua Y, Matsumoto K, Yamada A. Preventive and therapeutic effects in rats of hepatocyte growth factor infusion on liver fibrosis/cirrhosis. Hepatology 1997; 26: 81-9.

88. Ueki T, Kaneda Y, Tsutsui H. Hepatocyte growth factor gene therapy of liver cirrhosis in rats. Nat Med 1999; 5:22630 .

89. Yasuda H, Imai E, Shiota A, Fujise N, Morinaga T. Antifibrogenic effect of a deletion variant of hepatocyte growth factor on liver fibrosis in rat. Hepatology 1996; 24: 636-42.

90. Unemori EN, Pickford LB, Salles AL. Relaxin induces an extracellular matrix-degrading phenotype in human lung fibroblast in vitro and inhibits lung fibrosis in a murine model in vivo. J Clin Invest 1996; 98:2739-45.

91. Franklin TJ, Therapeutics approaches to organ fibrosis. Int J Biochem Cell Biol 1997; 29:79-89.

92. Windmeir C, Gressner AM. Effect of pentoxifylline on the fibrogenic functions of cultured rat liver fat storing 


\section{Tratamiento de la enfermedad hepática crónica}

cells and myofibroblasts. Biochem Pharmacol 1996; 51: 577-84.

93. Wu J, Zern MA. Hepatic stellate cells: a target for the treatment of liver fibrosis. J Gastroenterol. 2000; 35:66572.

94. Lee KS Cottam HB, Houglum K, Wasson DB, Carson D, Chojkier M. Pentoxifylline blocks hepatic stellate cell activation independently of phosphodiesterase inhibitory activity. Am J Physiol 1997; 273:G1094-100.

95. Desmouliaere A, Xu G Costa AM, Youset IM, Gabianni G. Effects of pentoxifylline on early proliferation and phenotypic modulation of fibrogenic cells in two rat model of fibrosis and on cultured hepatic stellate cells. J Hepatol 1999; 3:621-31.

96. Pinzani M, Gesualdo L, Sabbah GM, Abbound HE. Effects of platelet derived growth factor and other polypeptides mitogens on DNA synthesis and growth of cultured rat liver fat storing cells. J Clin Invest 1989; 84: 1786-93.

97. Marra F, Gentillini A, Pinzani M, Choudhury GG, Parola M, Herbst H. Phosphatidylinositol 3 kinase is required for platelet derived growth factor's actions on hepatic stellate cells. Gastroenterology 1997; 112:1297-306.

98. Marra F, Arriaghi MC, Fazi M, Caligiuri A, Pinzani M, Romanelli RG. Extracellular signal-regulated kinase activation differentially regulates platelet-derived growth factor's actions in hepatic stellate cells, and is induced by in vivo liver injury in the rat. Hepatology 1999; 30:951-8.

99. Arthur MJ, Iredali JP. Tissue inhibitor of metalloproteinases, hepatic stellate cells and liver fibrogenesis. J Gastroenterol Hepatol 1998; 13:S33-8.

100. Unemori EN, Pickfors LB, Salles AL. Relaxin induces an extracellular matriz-degrading phenotype in human lung fibroblast in vitro and inhibits lung fibrosis in amurine model in vivo. J Clin Invest 1996; 98:2739-45.

101. Prieto J, Quian C, Hernández-Alcoceba R, GonzálezAseguinolaza G, Mazzolini G, Sangro B, Kramer MG. Gene theraphy of liver diseases. Expert Opin Biol Ther 2004; 4: 1073-91.

102. Armendariz-Borunda J. Genomic medicine in Mexico. Applications of gene therapy for cirrosis reversion. Hepatol 2002; 1:169-74.
103. Chen M, Wang GJ, Diao Y, Xu RA, Xie HT, Xie HT, Li XY, Sun JG. Adeno-associated virus mediated interferongamma inhibits the progression of hepatic fibrosis in vitro and in vivo. World J Gastroentorol 2005; 11:4045-51.

104. Aurisicchio L, Delmastro P, Salucci V, Paz OG, Rovere P, Ciliberto G, et al. Liver-specific alpha 2 interferon gene expression results in protection from induced hepatitis. J Virol 2000; 74:4816-4823.

105. Hung KS, Lee TH, Chou WY, Wu CL, Cho CL, Lu CN, Jawan B, Wang CH. Interleukin-10 gene therapy reverses thioacetamide-induced liver fibrosis in mice. Bioche Biophys Res Commun 2005; 336:324-31.

106. Kronke J, Kittler R, Buchholz F, Windisch MP, Pietschmann T, Bartenschlager R, et al. Alternative approaches for efficient inhibition of hepatitis $C$ virus RNA replication by small interfering RNAs. J Virol 2004; 78: 3436-3446.

107. Chiou HC, Lucas MA, Coffin CC, Banaszczyk MG, Ill CR, Lollo CP. Gene therapy strategies for the treatment of chronic viral hepatitis. Expert Opin Biol Ther 2001; 1: 629-639.

108. Nakumuta M, Morizono S, Tsuruta S, Kohjima M, Kotoh K, Enjoji M. Remote delivery and expression of soluble type II TGF-beta receptor in muscle prevents hepatic fibrosis in rats. Int J Mol Med 2005; 16:59-64.

109. Lin Y, Xie WF, Chen YX, Zhang X, Zeng X, Qiang H, Chen WZ, Yang XJ, Han ZG, Zhang ZB. Treatment of experimental hepatic fibrosis by combinational delivery of urokinasetype plasminogen activator and hepatocyte growth genes. Liver Int 2005; 25:796-807. 